\title{
Effects of Galactic magnetic field on the UHECR anisotropy studies
}

\section{Ryo Higuchi, ${ }^{a, *}$ Takashi Sako, ${ }^{a}$ Kazumasa Kawata, ${ }^{a}$ Toshihiro Fujii ${ }^{b}$ and Eiji Kido ${ }^{c}$ on behalf of the the Telescope Array Collaboration}

(a complete list of authors can be found at the end of the proceedings)

${ }^{a}$ Institute for Cosmic Ray Research, University of Tokyo, Kashiwa, Chiba, Japan

${ }^{b}$ The Hakubi Center for Advanced Research and Graduate School of Science, Kyoto University, Kitashirakawa-Oiwakecho, Sakyo-ku, Kyoto, Japan

${ }^{c}$ Astrophysical Big Bang Laboratory, RIKEN,

Wako, Saitama, Japan

E-mail: rhiguchi@icrr.u-tokyo.ac.jp

Telescope Array (TA) and Auger experiments reported anisotropies in the arrival direction of ultrahigh-energy cosmic rays (UHECRs). In particular, Auger Collaboration reported a correlation between UHECR events and the flux model of assumed sources and suggested a contribution of starburst galaxies (SBGs) to the anisotropy of UHECRs. However, in their study, the effect of coherent deflections by the galactic magnetic field (GMF) is not taken into account. In this study, we investigated the effect of the GMF on the arrival directions of UHECRs using the cosmic ray propagation code CRPropa3. We used a backtracking technique which consists of propagating antiparticles to map the flux outside the galaxy to at the earth. We estimate the systematic effects caused by GMF in the reported likelihood analysis. We conduct likelihood analysis for mock UHECR datasets based on the flux pattern through the GMF model. We found systematic decrease of $\left(f_{\text {ani }}, \theta\right)$ due to GMF. As prospects for the TAx 4 experiment and joint analysis of Auger and TA collaborations, we develop the likelihood analysis method with the convolution of the rigidity spectrum.

$37^{\text {th }}$ International Cosmic Ray Conference (ICRC 2021)

July 12th-23rd, 2021

Online - Berlin, Germany

\footnotetext{
*Presenter
} 


\section{Introduction}

Understanding the origin of cosmic rays (CR) is one of the most important topics in astrophysics today. Telescope Array (TA) and Auger experiments report anisotropies in the arrival direction of ultra-high-energy cosmic rays (UHECRs) [1,2]. The anisotropies are important because the arrival direction of UHECR and their sources may be expected to correlate. In particular, the Auger collaboration reported a correlation between UHECR events and the flux model of assumed sources and suggested a contribution of starburst galaxies (SBGs) to the anisotropy of UHECRs [3, 4]. However, in their study, the effect of coherent deflections by the galactic magnetic field (GMF) is not taken into account. Due to the dependence of deflections by GMF on the rigidity $(R=E / Z e)$ of the particle, we need to take into account the continuous spectrum of rigidity of each UHECR. In this study, we estimate the systematic effects caused by GMF in reported likelihood analysis in $[3,4]$. As prospects for the TAx4 experiment and joint analysis of Auger and TA collaborations, we develop the new technique of likelihood analysis method with the convolution of the rigidity spectrum.

\section{Method}

In order to constrain the major source population of UHECRs, previous studies [3, 4] conducted a maximum-likelihood analysis. The maximum-likelihood analysis estimates two parameters, an anisotropic fraction $f_{\text {ani }}$ and a separation angular scale $\theta$. The anisotropic fraction $f_{\text {ani }}$ shows the fraction of all UHECR events due to the sources and the separation angular scale $\theta$ indicates the angular separation between an event and its source.

\subsection{Maximum-likelihood Analysis and Model CR Flux Pattern without coherent deflection by GMF}

We conduct the same maximum-likelihood analysis as in $[3,4]$. Test statistics $(T S)$ is written as

$$
T S=2 \ln \left(L\left(F_{\text {norm }}\right) / L\left(F_{\text {iso }}\right)\right)
$$

where $L(F)$ indicates a likelihood

$$
L(F)=\prod_{\mathrm{CR}} \frac{F\left(\mathbf{n}_{\mathrm{CR}}\right) * \omega\left(\mathbf{n}_{\mathrm{CR}}\right)}{\int_{4 \pi} F(\mathbf{n}) * \omega(\mathbf{n}) d \Omega} .
$$

where $F, \omega\left(\mathbf{n}_{\mathrm{CR}}\right)$ and $\mathbf{n}_{\mathrm{CR}}$ are a normalized model CR flux pattern, the exposure of each experiment and observed arrival directions of UHECRs, respectively. The normalized model CR flux pattern $F_{\text {norm }}$ can be written as convolution of a model CR flux pattern due to sources $F_{\text {org }}$ and isotropic flux pattern $F_{\text {iso }}$,

$$
\begin{array}{r}
F_{\text {norm }}\left(\mathbf{n}_{\mathrm{CR}}, f_{\text {ani }}, \theta\right)=f_{\text {ani }} * F_{\text {org }}^{\prime}\left(\mathbf{n}_{\mathrm{CR}}, \theta\right)+\left(1-f_{\text {ani }}\right) * F_{\text {iso }}, \\
F_{\text {org }}^{\prime}\left(\mathbf{n}_{\mathrm{CR}}, \theta\right)=\frac{F_{\text {org }}\left(\mathbf{n}_{\mathrm{CR}}, \theta\right)}{\int_{4 \pi} F_{\text {org }} d \Omega} \text { and } F_{\text {iso }}=1 / 4 \pi .
\end{array}
$$




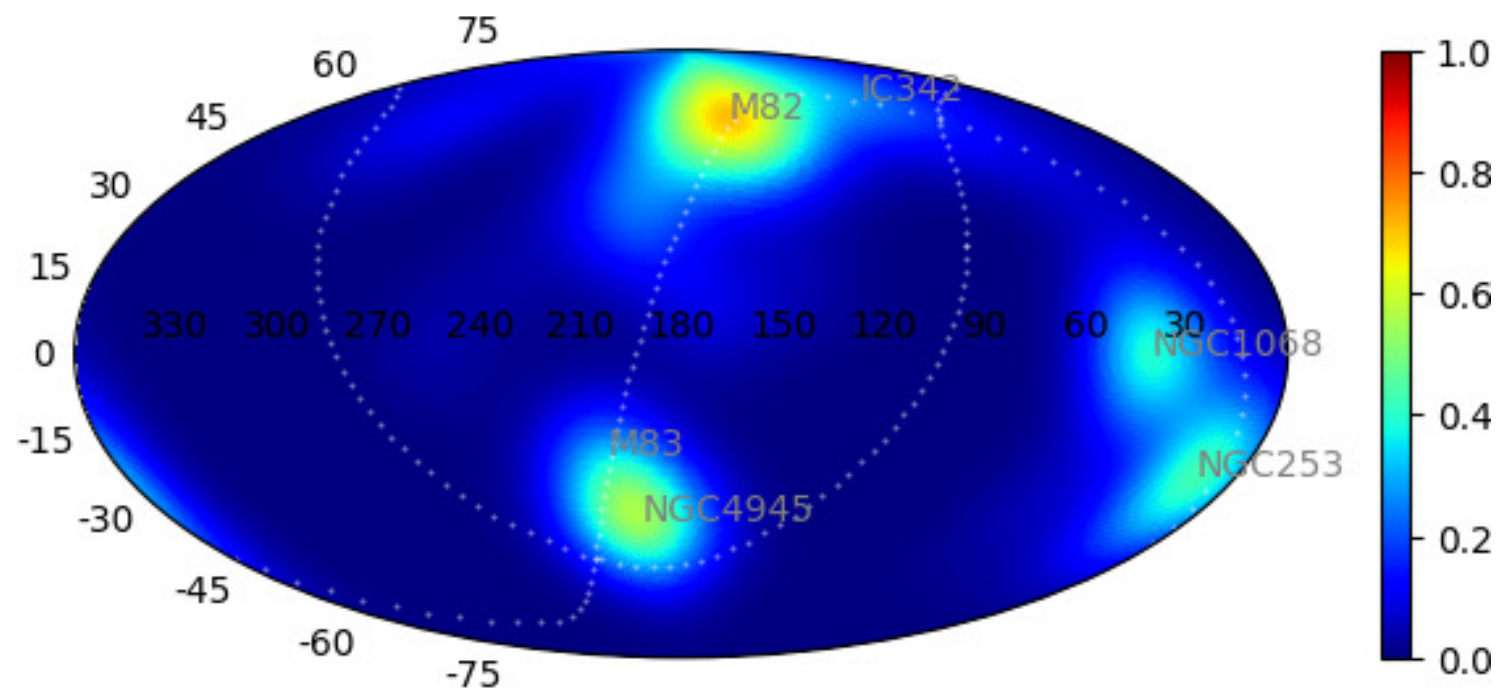

Figure 1: The original CR flux pattern from $\operatorname{SBGs} F_{\text {org }}(\mathbf{n}, \theta=13 \mathrm{deg}$ ) in equatorial coordinates (same as $[3,4])$.

Note that the exposure of each experiment $\omega\left(\mathbf{n}_{\mathrm{CR}}\right)$ varies with latitude of the experiment $\delta$ [5],

$$
\begin{array}{r}
\omega(\delta) \propto \cos \left(a_{0}\right) \cos (\delta) \sin \left(\alpha_{\mathrm{m}}\right)+\alpha_{\mathrm{m}} \sin \left(a_{0}\right) \sin (\delta) \\
\alpha_{\mathrm{m}}=\left\{\begin{array}{l}
0(\xi>1) \\
\pi(\xi<-1) \\
\cos ^{-1}(\xi)(-1<\xi<1)
\end{array}\right. \\
\xi=\frac{\cos \left(\theta_{\mathrm{m}}\right)-\sin \left(a_{0}\right) \sin (\delta)}{\cos \left(a_{0}\right) \cos (\delta)} .
\end{array}
$$

Refs. [3, 4] adopt CR flux pattern from SBGs $F_{\text {org }}(\mathbf{n}, \theta)$ as sum of von Mises - Fisher function of each source,

$$
F_{\text {org }}(\mathbf{n}, \theta)=\frac{\sum_{i} f_{i} \exp \left(\mathbf{n}_{i} \cdot \mathbf{n} / \theta^{2}\right)}{\int_{4 \pi} \sum_{i} f_{i} \exp \left(\mathbf{n}_{i} \cdot \mathbf{n} / \theta^{2}\right) d \Omega} .
$$

We refer to Table 1 in the previous analysis [3] for the values of $f_{i}$ and $\mathbf{n}_{i}$. An example of an original CR flux pattern $F_{\text {org }}(\mathbf{n}, \theta=13 \mathrm{deg})$ is shown in Figure 1. With assumption of the parameters $\left(f_{\text {ani }}, \theta\right)$, we estimate the best-fit parameters which maximizes the $T S$ in Equation 1. From the previous report by Auger Collaboration [3], the best-fit parameters by SBGs are estimated to be $\left(f_{\text {ani }}, \theta\right)=(9.7 \%, 12.9 \mathrm{deg})$.

\subsection{Backtracking Technique}

In order to account for the coherent deflection by GMF, we used a backtracking technique which propagates antiparticles from the earth. We use the cosmic ray propagation code CRPropa3 [6]. We adapt Jansson \& Farrar 2012 (JF12) model [7, 8] as GMF model. 

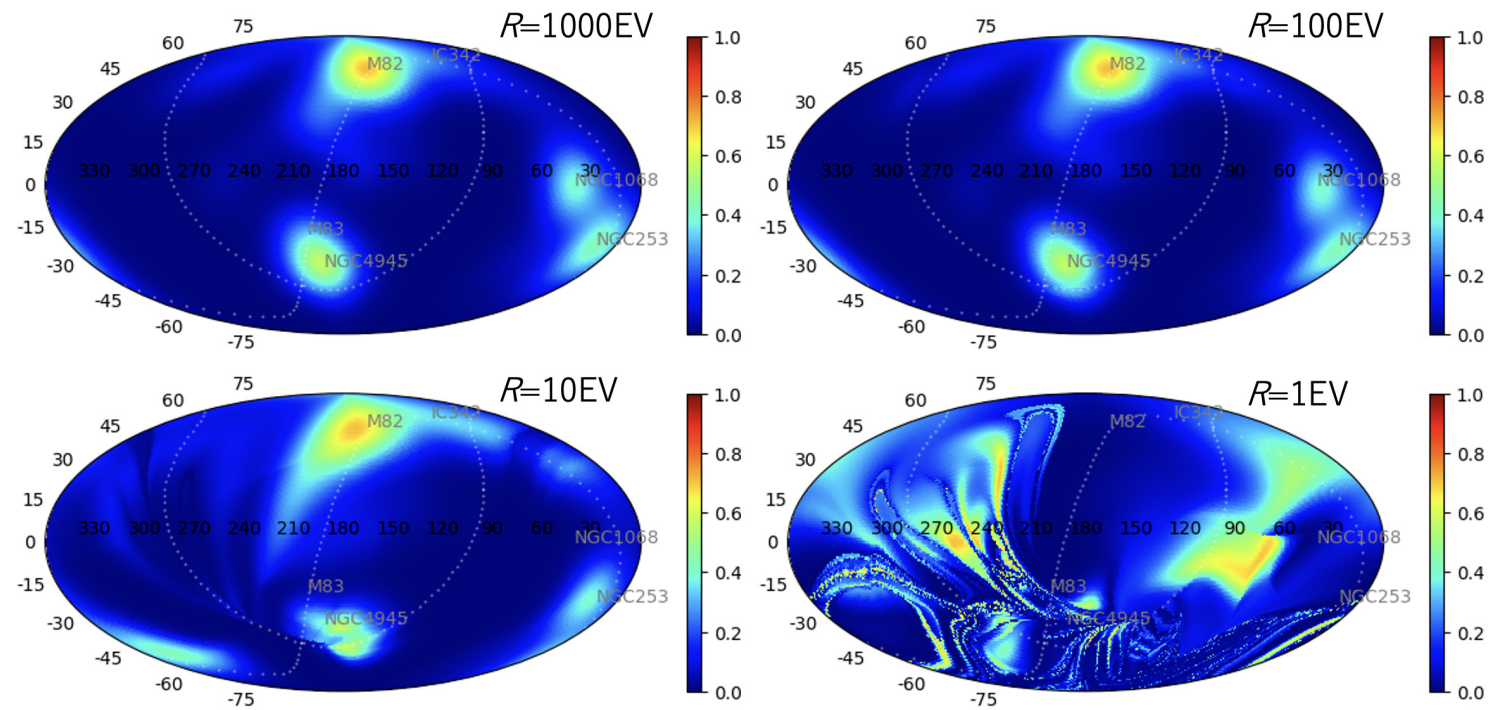

Figure 2: The the model CR flux pattern on the earth $F_{\text {earth }}\left(\mathbf{n}_{\text {earth }}, \theta=13 \mathrm{deg}, R\right)$ from SBGs in the case $R=1$ to $1000 \mathrm{EV}$ in equatorial coordinates.

\subsection{Modification of CR Flux Pattern}

We define the original CR flux pattern as $F_{\text {org }}\left(\mathbf{n}_{\text {org }}, \theta\right)$, where $\mathbf{n}_{\text {org }}$ show the direction at $20 \mathrm{kpc}$ from the galactic center (GC). We convert the directions on earth $\mathbf{n}_{\text {earth }}$ to those at $20 \mathrm{kpc}$ from the $\mathrm{GC} \mathbf{n}_{\text {org }}$ as

$$
\mathbf{n}_{\mathrm{org}}=A_{\mathrm{BT}}\left(\mathbf{n}_{\mathrm{earth}}, R\right) .
$$

We calculate the model CR flux pattern on the earth $F_{\text {earth }}$ as

$$
F_{\text {earth }}\left(\mathbf{n}_{\text {earth }}, \theta, R\right)=F_{\text {org }}\left(\mathbf{n}_{\text {org }}, \theta\right)=F_{\text {org }}\left(A_{\mathrm{BT}}\left(\mathbf{n}_{\text {earth }}, R\right), \theta\right),
$$

based on the Liouville's theorem. We calculate the CR flux pattern on the earth from $\log _{10}(R / \mathrm{EV})=$ 0.0 to $\log _{10}(R / \mathrm{EV})=3.0$ with 0.1 slices. We show examples of model CR flux pattern on the earth $F_{\text {earth }}$ in Figure 2.

\subsection{Mock UHECR datasets}

\subsubsection{Mock UHECR datasets without coherent deflection by GMF}

Based on the original model CR flux pattern in Section 2.1, we generate 1000 datasets each of which contains 4000 mock UHECR events.

\subsubsection{Mock UHECR datasets with coherent deflection by GMF}

Based on the modified flux pattern in Section 2.3, we generate 1000 datasets each of which contains 4000 mock UHECR events (all-sky data). We adopt a pure-proton composition $(Z=1)$ and a broken-power law spectrum in the spectral index with $\gamma=-2.69$ and $\gamma=-4.63$ broken at $e R=E=10^{19.81} \mathrm{eV}$ [9]. As an energy threshold $E_{\min }$ we use $E_{\min }=40 \mathrm{EeV}$ which correspond to $E_{\min }=39 \mathrm{EeV}$ in previous analysis [3]. 
Due to the discrete values of rigidity $R$ in the calculation of $F_{\text {earth }}$, we interpolate the flux value as

$$
F_{\text {earth }}\left(\mathbf{n}_{\text {earth }}, R\right)=(1-\alpha) * F_{\text {earth }}\left(\mathbf{n}_{\text {earth }}, R^{i}\right)+\alpha * F_{\text {earth }}\left(\mathbf{n}_{\text {earth }}, R^{i+1}\right),
$$

where $R^{i}$ is a discrete value of a rigidity of flux patterns $\left(\log _{10} R^{i}=0.0,0.1, \ldots, 3.0\right.$ for $i=$ $0,1, \ldots, 30)$ and

$$
\alpha=\frac{\log _{10} R-\log _{10} R^{i}}{\log _{10} R^{i+1}-\log _{10} R^{i}}\left(R^{i}<R<R^{i+1}\right) .
$$

\subsubsection{Selection of mock UHECR events based on the coverage of experiments}

For comparison with analysis for observed UHECR events [3, 4], we consider the coverage of TA and Auger experiment based on Equations 5 in Section 2.1. We adopt a latitude of an experiment $a_{0}=39.3(-35.2)$ and a maximum zenith angle $\theta_{\mathrm{m}}=55$ (60) for TA (Auger) experiment. Out of 4000 mock UHECR events in each dataset, approximately 1000 mock events are selected by the coverage of TA and Auger experiments. We define the datasets selected by the FoV of TA (Auger) experiment as north-sky (south-sky) datasets.

\section{Results}

In Sections 3.1 to 3.2, we apply the maximum-likelihood analysis of Section 2.1 to the mock UHECR datasets in Section 2.4. In Section 3.1, we apply the maximum-likelihood method to the mock UHECR datasets without coherent deflection by GMF. The results of the analysis of mock UHECR datasets with coherent deflection by GMF are shown in Section 3.2. In Section 3.3, we develop a new maxim-likelihood method which investigates a correlation between the model CR flux pattern on the earth $F_{\text {earth }}$ described in Section 2.3 and the mock UHECR events with coherent deflection by GMF constructed in Section 2.4.2.

\subsection{Analysis of mock events without GMF}

In the same manner as in Section 2.1, we calculate the TS of mock UHECRs of Section 2.4.1. We estimate the best-fit parameters of each dataset which maximizes the TS in Equation 1. We show an example of the results of likelihood analysis without GMF in Figure 3 (left). Black crosses in light color in Figure 3 show best-fit parameters for each all-sky dataset and blue circles and red triangles in light color indicate the best-fit parameters for each north-sky and south-sky dataset, respectively. We plot the median values of all best-fit parameters for all the 1000 datasets in thick colors. The median values of best-fit parameters for all-sky, north-sky, and south-sky datasets show the same distribution centered at the input (correct) parameters.

\subsection{Analysis of mock events with the inclusion of GMF}

We conduct the likelihood analysis with the mock UHECRs datasets of Section 2.4. We show an example of likelihood analysis including GMF in Figure 3 (right). We find a systematic decrease of $\left(f_{\text {ani }}, \theta\right)$ and discrepancy between north-sky datasets and all-sky, south-sky datasets. The systematic decrease in the north-sky is smaller than in the south-sky and all-sky due to the smaller deflection by GMF in the northern hemisphere. 

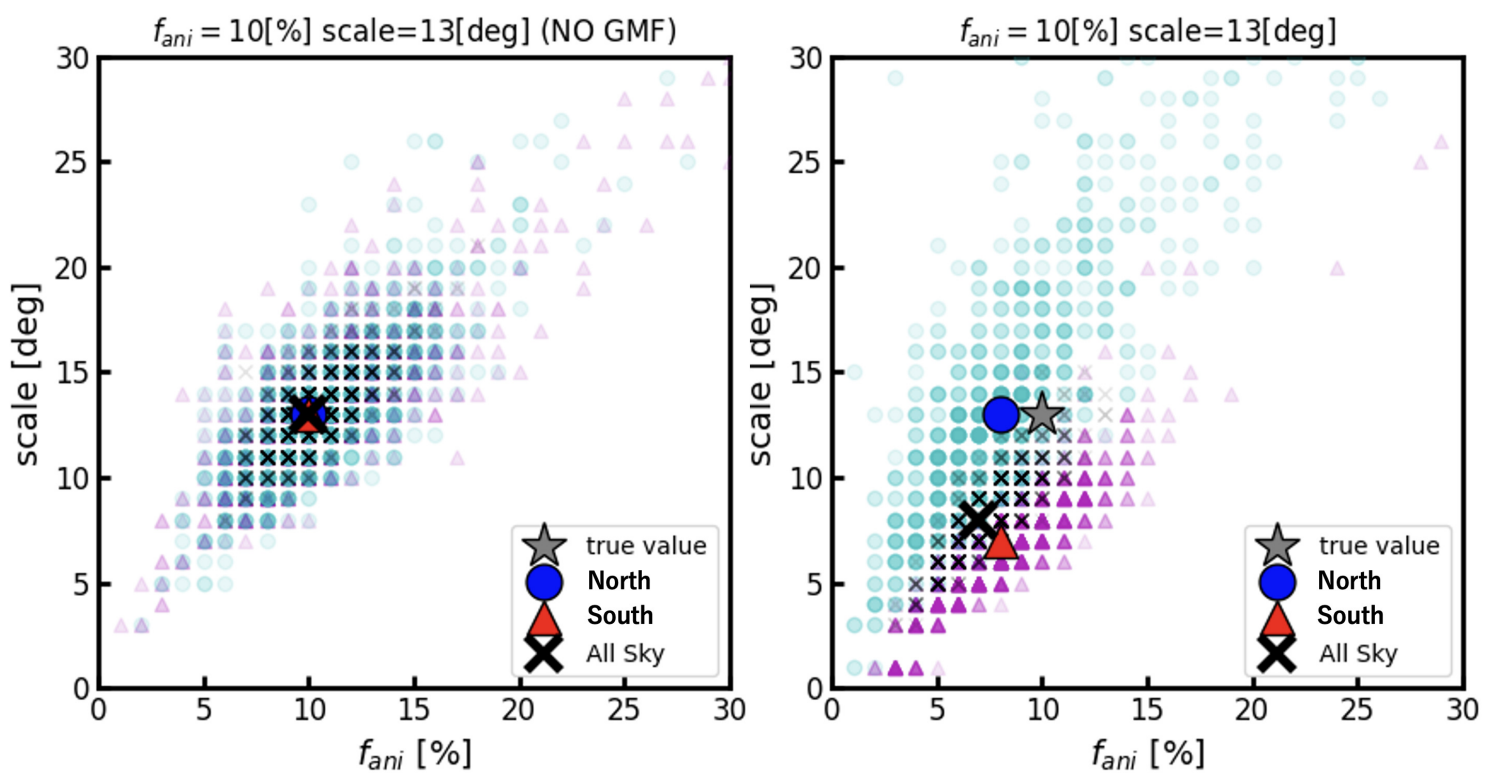

Figure 3: Examples of the results of likelihood analysis without GMF (left) and with GMF (right) in case of $\left(f_{\text {ani }}, \theta\right)=(10 \%, 13 \mathrm{deg})$. Black cross (crosses), blue circle (circles) and red triangle (triangles) in light (thick) color show the median value of the best-fit parameters of each (all the 1000) all-sky, north-sky and south-sky datasets, respectively.

\subsection{Development of Likelihood Analysis with Rigidity of UHECRs}

In order to reduce the systematic effects due to GMF, we develop a new likelihood analysis with the convolution of the rigidity spectrum. In a calculations of the likelihood (Equation 2) and $T S$ (Equation 1), we use the model CR flux pattern on the earth $F_{\text {earth }}$ instead of the original model CR flux pattern $F_{\text {org }}$ (Equation 3). We rewrite Equation 3 as follows:

$$
\begin{array}{r}
F_{\text {norm }}\left(\mathbf{n}, f_{\text {ani }}, \theta, R\right)=f_{\text {ani }} * F_{\text {earth }}^{\prime}(\mathbf{n}, \theta, R)+\left(1-f_{\text {ani }}\right) * F_{\text {iso }}, \\
F_{\text {earth }}^{\prime}=\frac{F_{\text {earth }}(\mathbf{n}, \theta, R)}{\int_{4 \pi} F_{\text {earth }} d \Omega}
\end{array}
$$

We show the result of likelihood analysis with $F_{\text {earth }}$ in the left panel of Figure 4. The systematic effect caused by GMF can be reduced.

\section{Summary}

Telescope Array (TA) and Auger experiments reported anisotropies in the arrival direction of ultra-high-energy cosmic rays (UHECRs). In particular, Auger Collaboration reported a correlation between UHECR events and the flux model of assumed sources, and suggested a contribution of starburst galaxies (SBGs) to the anisotropy of UHECRs. However, in their study, the effect of coherent deflections by the galactic magnetic field (GMF) is not taken into account. We estimate the systematic effects caused by GMF in reported likelihood analysis in [3, 4]. We conduct likelihood analysis for mock UHECR datasets based on the flux pattern processed through GMF model. We found systematic decrease of $\left(f_{\text {ani }}, \theta\right)$ due to GMF. As prospects for the TAx4 experiment and 


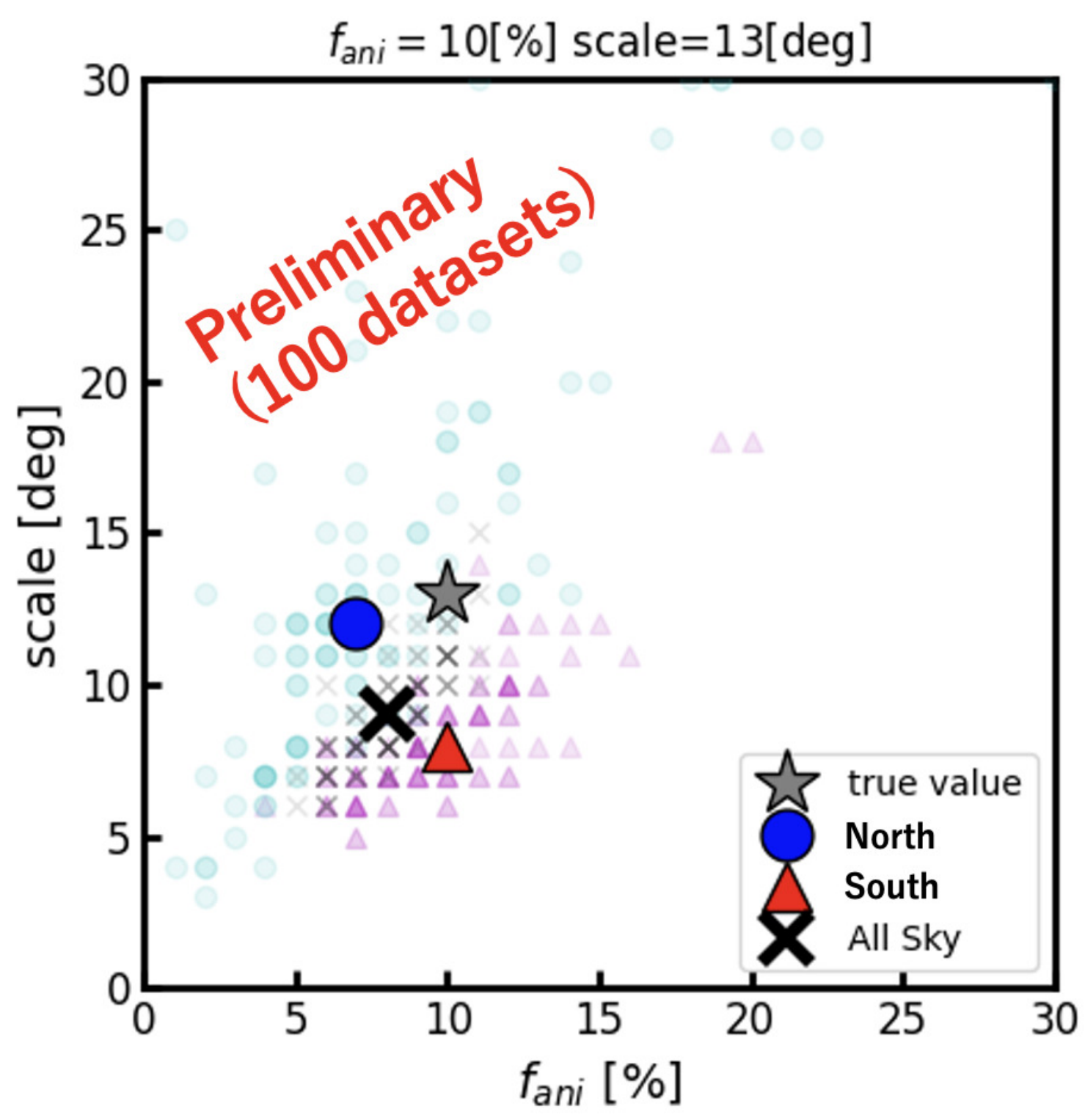

Figure 4: Same as the left panel in Figure 3, but that of likelihood analysis with $F_{\text {earth }}$ in case of $\left(f_{\text {ani }}, \theta\right)=$ $(10 \%, 13 \mathrm{deg})$ for 100 datasets.

joint analysis of Auger and TA collaborations, we develop the likelihood analysis method with the convolution of the rigidity spectrum.

\section{References}

[1] R. U. Abbasi et al. Indications of intermediate-scale anisotropy of cosmic rays with energy greater than 57 eev in the northern sky measured with the surface detector of the telescope array experiment. Astrophysical Journal Letters, 790, 2014. 
[2] A. Aab et al. Searches for anisotropies in the arrival directions of the highest energy cosmic rays detected by the pierre auger observatory. Astrophysical Journal, 804, 2015.

[3] A. Aab et al. An indication of anisotropy in arrival directions of ultra-high-energy cosmic rays through comparison to the flux pattern of extragalactic gamma-ray sources. The Astrophysical Journal, 853:L29, 2018.

[4] R. U. Abbasi et al. Testing a reported correlation between arrival directions of ultra-high-energy cosmic rays and a flux pattern from nearby starburst galaxies using telescope array data. The Astrophysical Journal, 867:L27, 2018.

[5] Paul Sommers. Cosmic ray anisotropy analysis with a full-sky observatory. Astroparticle Physics, 14:271-286, 2001.

[6] Rafael Alves Batista et al. Crpropa 3 - a public astrophysical simulation framework for propagating extraterrestrial ultra-high energy particles. Journal of Cosmology and Astroparticle Physics, 2016, 2016.

[7] Ronnie Jansson and Glennys R. Farrar. A new model of the galactic magnetic field. Astrophysical Journal, 757, 2012.

[8] Ronnie Jansson and Glennys R. Farrar. The galactic magnetic field. Astrophysical Journal Letters, 761:1-5, 2012.

[9] Yoshiki Tsunesada, Tareq Abuzayyad, Dmitri Ivanov, and Gordon Thomson. Measured by the telescope array. pages 4-11, 2017.

\section{Full Authors List: the Telescope Array Collaboration}

R.U. Abbasi ${ }^{1,2}$, T. Abu-Zayyad ${ }^{1,2}$, M. Allen ${ }^{2}$, Y. Arai $^{3}$, R. Arimura ${ }^{3}$, E. Barcikowski², J.W. Belz ${ }^{2}$, D.R. Bergman ${ }^{2}$, S.A. Blake ${ }^{2}$, I. Buckland ${ }^{2}$, R. Cady $^{2}$, B.G. Cheon ${ }^{4}$, J. Chiba ${ }^{5}$, M. Chikawa ${ }^{6}$, T. Fujii ${ }^{7}$, K. Fujisue ${ }^{6}$, K. Fujita ${ }^{3}$, R. Fujiwara ${ }^{3}$, M. Fukushima ${ }^{6}$, R. Fukushima ${ }^{3}$, G. Furlich ${ }^{2}$, R. Gonzalez ${ }^{2}$, W. Hanlon ${ }^{2}$, M. Hayashi ${ }^{8}$, N. Hayashida ${ }^{9}$, K. Hibino ${ }^{9}$, R. Higuchi ${ }^{6}$, K. Honda ${ }^{10}$, D. Ikeda ${ }^{9}$, T. Inadomi ${ }^{11}$, N. Inoue ${ }^{12}$, T. Ishii ${ }^{10}$, H. Ito ${ }^{13}$, D. Ivanov ${ }^{2}$, H. Iwakura ${ }^{11}$, A. Iwasaki ${ }^{3}$, H.M. Jeong ${ }^{14}$, S. Jeong ${ }^{14}$, C.C.H. Jui ${ }^{2}$, K. Kadota ${ }^{15}$, F. Kakimoto ${ }^{9}$, O. Kalashev ${ }^{16}$, K. Kasahara ${ }^{17}$, S. Kasami ${ }^{18}$, H. Kawai ${ }^{19}$, S. Kawakami ${ }^{3}$, S. Kawana ${ }^{12}$, K. Kawata ${ }^{6}$, I. Kharuk ${ }^{16}$, E. Kido ${ }^{13}$, H.B. Kim ${ }^{4}$, J.H. Kim ${ }^{2}$, J.H. Kim ${ }^{2}$, M.H. Kim ${ }^{14}$, S.W. Kim ${ }^{14}$, Y. Kimura ${ }^{3}$, S. Kishigami ${ }^{3}$, Y. Kubota ${ }^{11}$, S. Kurisu ${ }^{11}$, V. Kuzmin ${ }^{161}$, M. Kuznetsov ${ }^{16,20}$, Y.J. Kwon ${ }^{21}$, K.H. Lee ${ }^{14}$, B. Lubsandorzhiev ${ }^{16}$, J.P. Lundquist ${ }^{2,22}$, K. Machida ${ }^{10}$, H. Matsumiya ${ }^{3}$, T. Matsuyama ${ }^{3}$, J.N. Matthews ${ }^{2}$, R. Mayta ${ }^{3}$, M. Minamino ${ }^{3}$, K. Mukai ${ }^{10}$, I. Myers ${ }^{2}$, S. Nagataki ${ }^{13}$, K. Nakai ${ }^{3}$, R. Nakamura ${ }^{11}$, T. Nakamura ${ }^{23}$, T. Nakamura ${ }^{11}$, Y. Nakamura ${ }^{11}$, A. Nakazawa ${ }^{11}$, E. Nishio ${ }^{18}$, T. Nonaka ${ }^{6}$, H. Oda ${ }^{3}$, S. Ogio ${ }^{3,24}$, M. Ohnishi ${ }^{6}$, H. Ohoka ${ }^{6}$, Y. Oku ${ }^{18}$, T. Okuda ${ }^{25}$, Y. Omura ${ }^{3}$, M. Ono ${ }^{13}$, R. Onogi ${ }^{3}$, A. Oshima ${ }^{3}$, S. Ozawa ${ }^{26}$, I.H. Park ${ }^{14}$, M. Potts ${ }^{2}$, M.S. Pshirkov ${ }^{16,27}$, J. Remington ${ }^{2}$, D.C. Rodriguez ${ }^{2}$, G.I. Rubtsov ${ }^{16}$, D. Ryu ${ }^{28}$, H. Sagawa ${ }^{6}$, R. Sahara ${ }^{3}$, Y. Saito ${ }^{11}$, N. Sakaki ${ }^{6}$, T. Sako ${ }^{6}$, N. Sakurai ${ }^{3}$, K. Sano ${ }^{11}$, K. Sato ${ }^{3}$, T. Seki ${ }^{11}$, K. Sekino ${ }^{6}$, P.D. Shah ${ }^{2}$, Y. Shibasaki ${ }^{11}$, F. Shibata ${ }^{10}$, N. Shibata ${ }^{18}$, T. Shibata ${ }^{6}$, H. Shimodaira ${ }^{6}$, B.K. Shin ${ }^{28}$, H.S. Shin ${ }^{6}$, D. Shinto ${ }^{18}$, J.D. Smith ${ }^{2}$, P. Sokolsky ${ }^{2}$, N. Sone ${ }^{11}$, B.T. Stokes ${ }^{2}$, T.A. Stroman ${ }^{2}$, Y. Takagi ${ }^{3}$,

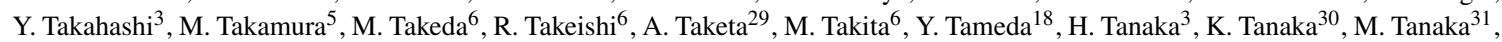
Y. Tanoue ${ }^{3}$, S.B. Thomas ${ }^{2}$, G.B. Thomson ${ }^{2}$, P. Tinyakov ${ }^{16,20}$, I. Tkachev ${ }^{16}$, H. Tokuno ${ }^{32}$, T. Tomida ${ }^{11}$, S. Troitsky ${ }^{16}$, R. Tsuda ${ }^{3}$, Y. Tsunesada ${ }^{3,24}$, Y. Uchihori ${ }^{33}$, S. Udo ${ }^{9}$, T. Uehama ${ }^{11}$, F. Urban ${ }^{34}$, T. Wong ${ }^{2}$, K. Yada $^{6}$, M. Yamamoto ${ }^{11}$, K. Yamazaki ${ }^{9}$, J. Yang ${ }^{35}$, K. Yashiro ${ }^{5}$, F. Yoshida ${ }^{18}$, Y. Yoshioka ${ }^{11}$, Y. Zhezher ${ }^{6,16}$, and Z. Zundel ${ }^{2}{ }^{1}$ Department of Physics, Loyola University Chicago, Chicago, Illinois, USA

${ }^{2}$ High Energy Astrophysics Institute and Department of Physics and Astronomy, University of Utah, Salt Lake City, Utah, USA

${ }^{3}$ Graduate School of Science, Osaka City University, Osaka, Osaka, Japan

${ }^{4}$ Department of Physics and The Research Institute of Natural Science, Hanyang University, Seongdong-gu, Seoul, Korea

${ }^{5}$ Department of Physics, Tokyo University of Science, Noda, Chiba, Japan

${ }^{6}$ Institute for Cosmic Ray Research, University of Tokyo, Kashiwa, Chiba, Japan 
${ }^{7}$ The Hakubi Center for Advanced Research and Graduate School of Science, Kyoto University, Kitashirakawa-Oiwakecho, Sakyo-ku, Kyoto, Japan

${ }^{8}$ Information Engineering Graduate School of Science and Technology, Shinshu University, Nagano, Nagano, Japan

${ }^{9}$ Faculty of Engineering, Kanagawa University, Yokohama, Kanagawa, Japan

${ }^{10}$ Interdisciplinary Graduate School of Medicine and Engineering, University of Yamanashi, Kofu, Yamanashi, Japan

${ }^{11}$ Academic Assembly School of Science and Technology Institute of Engineering, Shinshu University, Nagano, Nagano, Japan

12 The Graduate School of Science and Engineering, Saitama University, Saitama, Saitama, Japan

${ }^{13}$ Astrophysical Big Bang Laboratory, RIKEN, Wako, Saitama, Japan

${ }^{14}$ Department of Physics, SungKyunKwan University, Jang-an-gu, Suwon, Korea

${ }^{15}$ Department of Physics, Tokyo City University, Setagaya-ku, Tokyo, Japan

${ }^{16}$ Institute for Nuclear Research of the Russian Academy of Sciences, Moscow, Russia

${ }^{17}$ Faculty of Systems Engineering and Science, Shibaura Institute of Technology, Minato-ku, Tokyo, Japan

${ }^{18}$ Department of Engineering Science, Faculty of Engineering, Osaka Electro-Communication University, Neyagawa-shi, Osaka, Japan

${ }^{19}$ Department of Physics, Chiba University, Chiba, Chiba, Japan

${ }^{20}$ Service de Physique Th??orique, Universit?? Libre de Bruxelles, Brussels, Belgium

${ }^{21}$ Department of Physics, Yonsei University, Seodaemun-gu, Seoul, Korea

${ }^{22}$ Center for Astrophysics and Cosmology, University of Nova Gorica, Nova Gorica, Slovenia

${ }^{23}$ Faculty of Science, Kochi University, Kochi, Kochi, Japan

${ }^{24}$ Nambu Yoichiro Institute of Theoretical and Experimental Physics, Osaka City University, Osaka, Osaka, Japan

${ }^{25}$ Department of Physical Sciences, Ritsumeikan University, Kusatsu, Shiga, Japan

${ }^{26}$ Quantum ICT Advanced Development Center, National Institute for Information and Communications Technology, Koganei, Tokyo, Japan

${ }^{27}$ Sternberg Astronomical Institute, Moscow M.V. Lomonosov State University, Moscow, Russia

${ }^{28}$ Department of Physics, School of Natural Sciences, Ulsan National Institute of Science and Technology, UNIST-gil, Ulsan, Korea

${ }^{29}$ Earthquake Research Institute, University of Tokyo, Bunkyo-ku, Tokyo, Japan

${ }^{30}$ Graduate School of Information Sciences, Hiroshima City University, Hiroshima, Hiroshima, Japan

${ }^{31}$ Institute of Particle and Nuclear Studies, KEK, Tsukuba, Ibaraki, Japan

${ }^{32}$ Graduate School of Science and Engineering, Tokyo Institute of Technology, Meguro, Tokyo, Japan

${ }_{33}$ Department of Research Planning and Promotion, Quantum Medical Science Directorate, National Institutes for Quantum and Radiological Science and Technology, Chiba, Chiba, Japan

${ }^{34}$ CEICO, Institute of Physics, Czech Academy of Sciences, Prague, Czech Republic

${ }^{35}$ Department of Physics and Institute for the Early Universe, Ewha Womans University, Seodaaemun-gu, Seoul, Korea 